\title{
In vitro effects of purine and pyrimidine analogues on Leishmania donovani and Leishmania infantum promastigotes and intracellular amastigotes
}

\author{
Samira Azzouz ${ }^{1,2}$ and Philippe Lawton ${ }^{1,2 *}$ \\ ${ }^{1}$ Université de Lyon, Université Claude-Bernard Lyon I, ISPB-Faculté de Pharmacie, Lyon, France; \\ ${ }^{2}$ Institut de recherche pour le développement (IRD), UMR InterTryp IRD/CIRAD, campus international de Baillarguet, Montpellier, France
}

\begin{abstract}
Inhibition of parasite metabolic pathways is a rationale for new chemotherapeutic strategies. The pyrimidine and purine salvage pathways are thus targets against Leishmania donovani and L. infantum, causative agents of visceral human leishmaniasis and canine leishmaniosis. The antiproliferative effect of the pyrimidine analogues Cytarabine and 5-fluorouracil and of the purine analogues Azathioprine and 6-mercaptopurine was evaluated in vitro on the promastigote and the intracellular amastigote stages of the parasite. Cytarabine and 5-fluorouracil were the best inhibitors against promastigotes, whereas 5- fluorouracil and azathioprine displayed the best efficacy against the amastigote stage. The ultrastructural study showed an important cytoplasmic vacuolization and with azathioprine and 5-fluorouracyl, a mitochondrial swelling and appearance of autophagosome-like structures. Alterations of the kinetoplast were also observed with 5-fluorouracil, all these damages eventually resulting in an autolysis process that triggered the subsequent death of the intracellular parasites.
\end{abstract}

\section{Keywords}

L. donovani, L. infantum, purine analogues, pyrimidine analogues, antiproliferative effect, ultrastructural modifications

\section{Introduction}

Leishmaniasis is a disease caused by a number of species of protozoa of the genus Leishmania. Of the four major clinical types of this infection, visceral leishmaniasis or Kala-azar caused in humans by L. donovani and human visceral and canine leishmaniosis caused by L. chagasi/infantum are the most severe. The first-line therapies against visceral leishmaniasis are the two pentavalent antimonial compounds, sodium stibogluconate (Pentostam) and meglumine antimoniate (Glucantime), while pentamidine isethionate and amphotericin B desoxycholate are the two second-line agents (Monge-Maillo and LópezVélez 2013, Sundar and Chakravarty 2013). Among their numerous drawbacks, they require parenteral administration, they have significant toxic side effects and widespread resistance to them has been reported mainly in India, Kenya and Sudan (Croft and Olliaro 2011). Despite its effectiveness and its safety, liposomal amphotericin B is costly (Balasegaram et al. 2012). The development of the parasite's resistance to current drugs and with the exception of liposomal amphotericin B, their lack of efficacy against VL/HIV co-infections has emphasized the need for new drugs. Miltefosine inhibits the phospholipid and sterol biosynthesis in Trypanosomatids and is effective in vivo against Leishmania, even by the oral route. However, its long half-life and its teratogenic potential are important drawbacks, as development of drug resistance may eventually lead to a premature inefficacy of this very important drug (Dorlo et al. 2012, Pachioni et al. 2013).

A rational discovery of novel antiparasitic drugs should be based on parasite-specific metabolisms (Vincent and Barrett 2015). Pyrimidine biosynthesis is a vital biological process, achieved both by de novo synthesis and by pyrimidine salvage pathways and enzymes of this pathway are considered as potential drug targets. Like most parasitic protozoa, Leishmania is also an obligate purine auxotroph and hence depends on its own purine salvage pathway that has also been considered as a target for novel treatments against leishmaniasis. The purine analogue allopurinol alone or in combination with other drugs has been proven to be effective against canine leishmaniosis (Farca et al. 2012), but its pharmacokinetic properties are a major limitation to its use for treatment of human leishmaniasis (Croft et al. 2006). 
In the present work, we tested drugs used against other diseases in humans, two purine analogues - 6-mercaptopurine and azathioprine - and two pyrimidine derivatives - cytarabine and 5-fluorouracil - on the promastigote and amastigote stages of $L$. donovani and $L$. infantum, two agents of visceral diseases that exhibit a clinical polymorphism. As a purine analogue and a drug used against canine leishmaniosis, allopurinol was used as a control.

\section{Materials and Methods}

\section{Drugs}

The control drugs Glucantime and allopurinol, the pyrimidine analogues 5-fluorouracil, cytarabine (Cytosine $\beta$-D-arabinofuranoside) and the purine analogues azathioprine and 6-mercaptopurine were purchased from Sigma Aldrich (St. Quentin Fallavier, France). They were dissolved in DMSO and $1 \mathrm{mM}$ stock solutions were made extemporaneously by dilution with culture medium. Serial dilutions ranging from 0.1 to $100 \mu \mathrm{M}$ were performed in the relevant culture media.

\section{Cytotoxicity tests}

Mouse peritoneal macrophages J774A.1 (ECACC, 91051511) were cultured in RPMI 1640 (Sigma Aldrich, St. Quentin Fallavier, France). When the cells had developed a monolayer, they were incubated in 96-well plates for 4 hours with $100 \mu \mathrm{L}$ of the drugs at concentrations ranging from 0.1 to $100 \mu \mathrm{M}$ with a maximum of $0.1 \%(\mathrm{v} / \mathrm{v})$ DMSO. The cell viability was assessed after $24 \mathrm{hr}, 48 \mathrm{hr}$ and $72 \mathrm{hr}$ using a methyl-tetrazolium salt (MTS)/phenazine methosulphate (PMS) solution (CellTiter 96 ${ }^{\circledR}$, Promega, Madison, WI, USA). The absorbance of the formazan product was measured at 490 nm with a Multiskan EX plate reader (Thermo Fisher Scientific, Villebon sur Yvette, France). The $\mathrm{IC}_{50}$ values were calculated from the drug concentration-response curves.

\section{Promastigote culture and assays}

The Leishmania donovani strain LCR-133 (Leishmania Reference Center, Jerusalem, Israel) used in this study was isolated in 1967 from a human case of Kala-azar in Behenber (Ethiopia) and the L. infantum strain was isolated from an infected dog from Spain. The promastigotes were grown in 75 $\mathrm{cm}^{2}$ culture flasks at $22^{\circ} \mathrm{C}$ in TC-199 medium supplemented with $20 \%$ heat-inactivated foetal bovine serum and $2 \%$ penicillin-streptomycin (PAA, Vélizy Villacoublay, France). At the end of the exponential growth phase, the cultures were centrifuged at $1500 \mathrm{rpm}$ for $15 \mathrm{~min}$, the promastigotes were diluted at $1 \times 10^{6}$ parasites $/ \mathrm{ml}$ and $200 \mu \mathrm{l}$ of the suspension were distributed in duplicates into 96 multi-well plates with the analogues at final concentrations ranging from 0.1 to $100 \mu \mathrm{M}$. The inhibition of promastigote growth was deter- mined with a Leica DM4000B microscope at a $\times 100$ magnification using a Malassez haemocytometer after 4 and $24 \mathrm{hr}$. The growth inhibition was calculated using the following formula: $\mathrm{I}=100-[\mathrm{T} / \mathrm{C} \times 100]$, where $\mathrm{I}=$ percentage of inhibition, $\mathrm{C}=$ number of parasites in the control, $\mathrm{T}=$ number of parasites in the treated wells.

\section{Amastigote assay}

To test the activity of the nucleoside analogues on the intracellular amastigote stage of the parasite, J774A.1 macrophages were cultured in triplicates on sterile glass coverslips in 24-well plates at a mean cell density of 75,000 cells/well. After adhesion of the cells, the medium was removed and replaced with fresh medium containing the Leishmania promastigotes at a 10 parasites per cell ratio. After $16 \mathrm{hrs}$ at $37^{\circ} \mathrm{C}$, the medium was removed, the infected cultures were washed 3 times with serum-free RPMI medium and incubated for 4 hrs with the various compounds at final concentrations ranging from 1 to $50 \mu \mathrm{M}$. The cultures were then washed with fresh medium and subsequently allowed to grow for 24,48 and $72 \mathrm{hrs}$. The coverslips were removed, fixed with methanol and stained with Giemsa. The intracellular amastigotes were counted by light microscopy and the percentage of inhibition was calculated as above. A minimum of 500 cells was counted in each replicate. Control cultures were infected as described above but without the drugs. All the experiments were repeated at least 3 times.

\section{Statistical analysis}

The Prism ${ }^{\circledR} 6$ software (Graph Pad Software, Berkeley, CA, USA) was used to calculate the half maximum inhibitory concentrations $\left(\mathrm{IC}_{50}\right)$ by nonlinear regression and to evaluate the statistical significance of the growth inhibition.

\section{Transmission electron microscopy (TEM) of infected macrophages}

After $24 \mathrm{hr}$, control and treated cultures were fixed with 4\% glutaraldehyde in $0.2 \mathrm{M}$ cacodylate buffer, $\mathrm{pH} 7.4$, post-fixed with $1 \%$ osmium tetroxide and $0.8 \%$ potassium ferrocyanide in $0.1 \mathrm{M}$ cacodylate buffer, dehydrated in a graded series of acetone and embedded in Epon resin. Ultrathin sections were stained with uranyl acetate and lead citrate and observed with a Jeol 1400 JEM transmission electron microscope (Tokyo, Japan) set at $80 \mathrm{kV}$.

\section{Results}

\section{Cytotoxicity}

The cell viability decreased proportionally with the time of incubation with Amphotericin B, the only toxic control drug and 


\section{$4 \mathrm{hr}$}

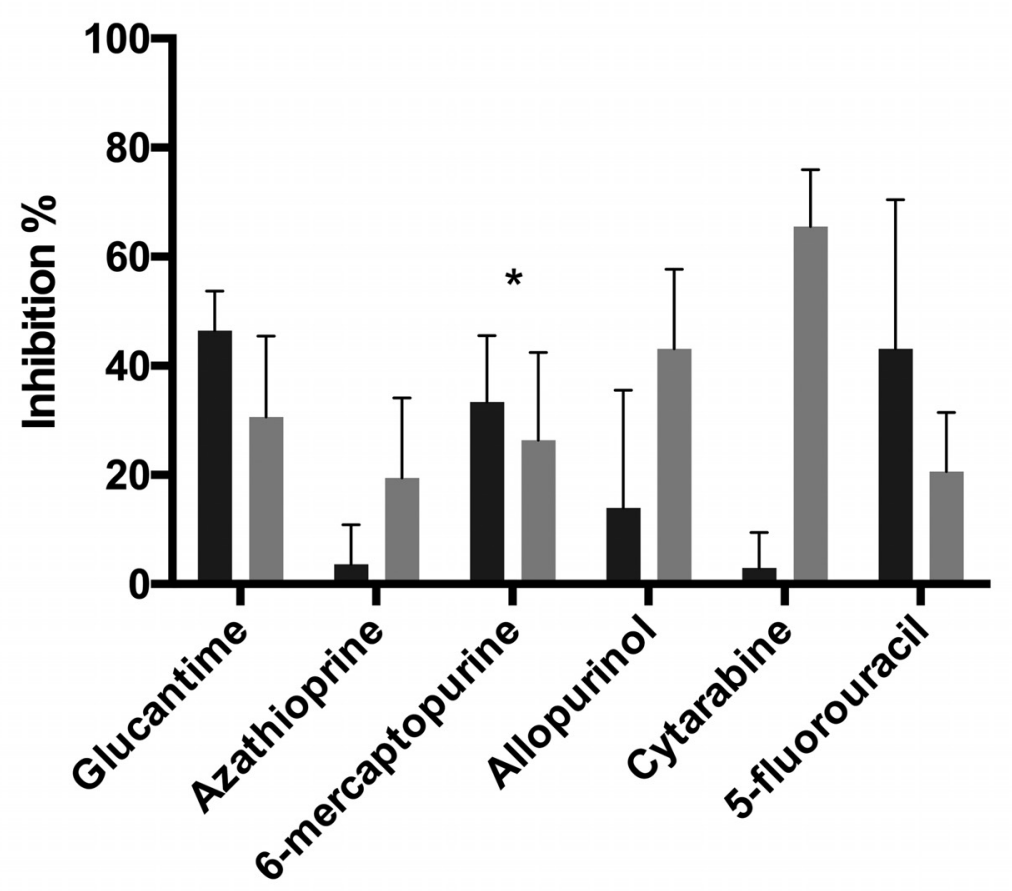

L. donovani
L. infantum

\section{$24 \mathrm{hr}$}

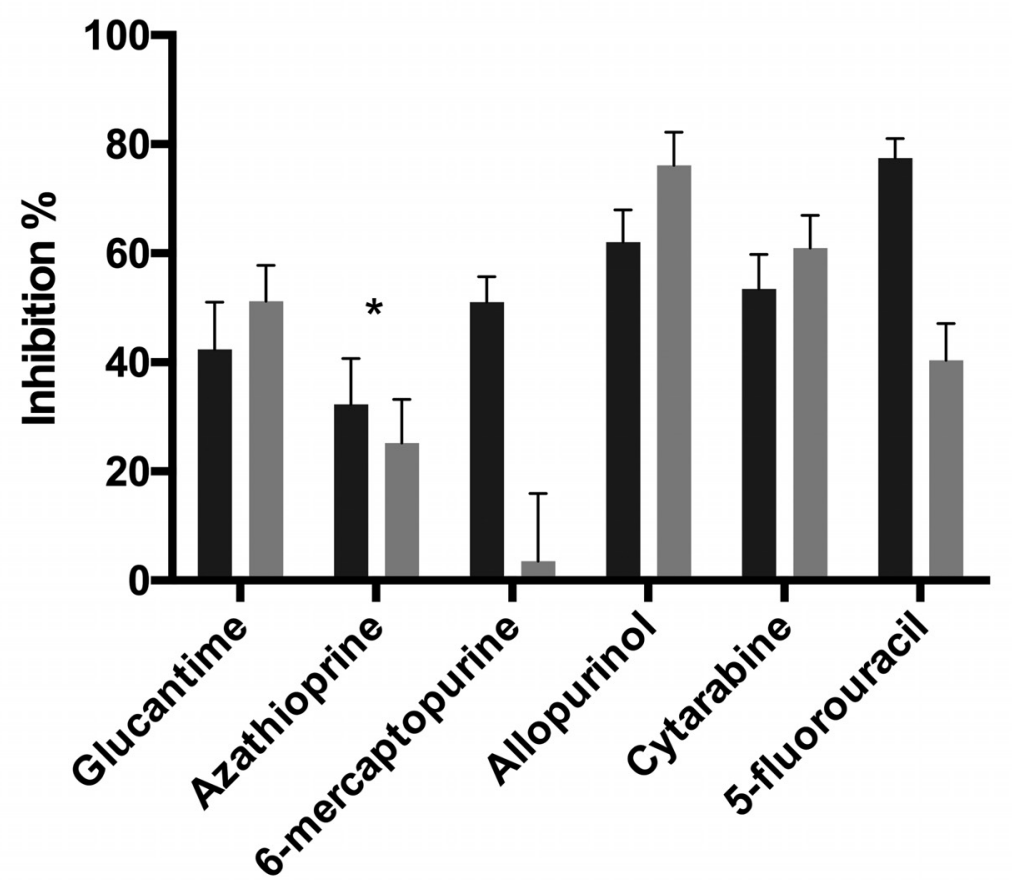

Fig. 1. Example of the differential inhibitory effects of the analogues $(50 \mu \mathrm{M})$ on $L$. donovani and L. infantum promastigotes. The parasites at a starting concentration of $0.8 \times 10^{6} / \mathrm{ml}$ were incubated for 4 and $24 \mathrm{hrs}$ with the nucleoside analogues. The final parasite concentrations in the control wells were $2.1 \pm 0.23 \times 10^{6} / \mathrm{ml}$ for $L$. donovani and $0.93 \pm 0.1 \times 10^{6} / \mathrm{ml}$ for $L$. infantum. Results of three separate experiments. *: no significant difference $(p>0.05)$ between the L. donovani and L. infantum strains 

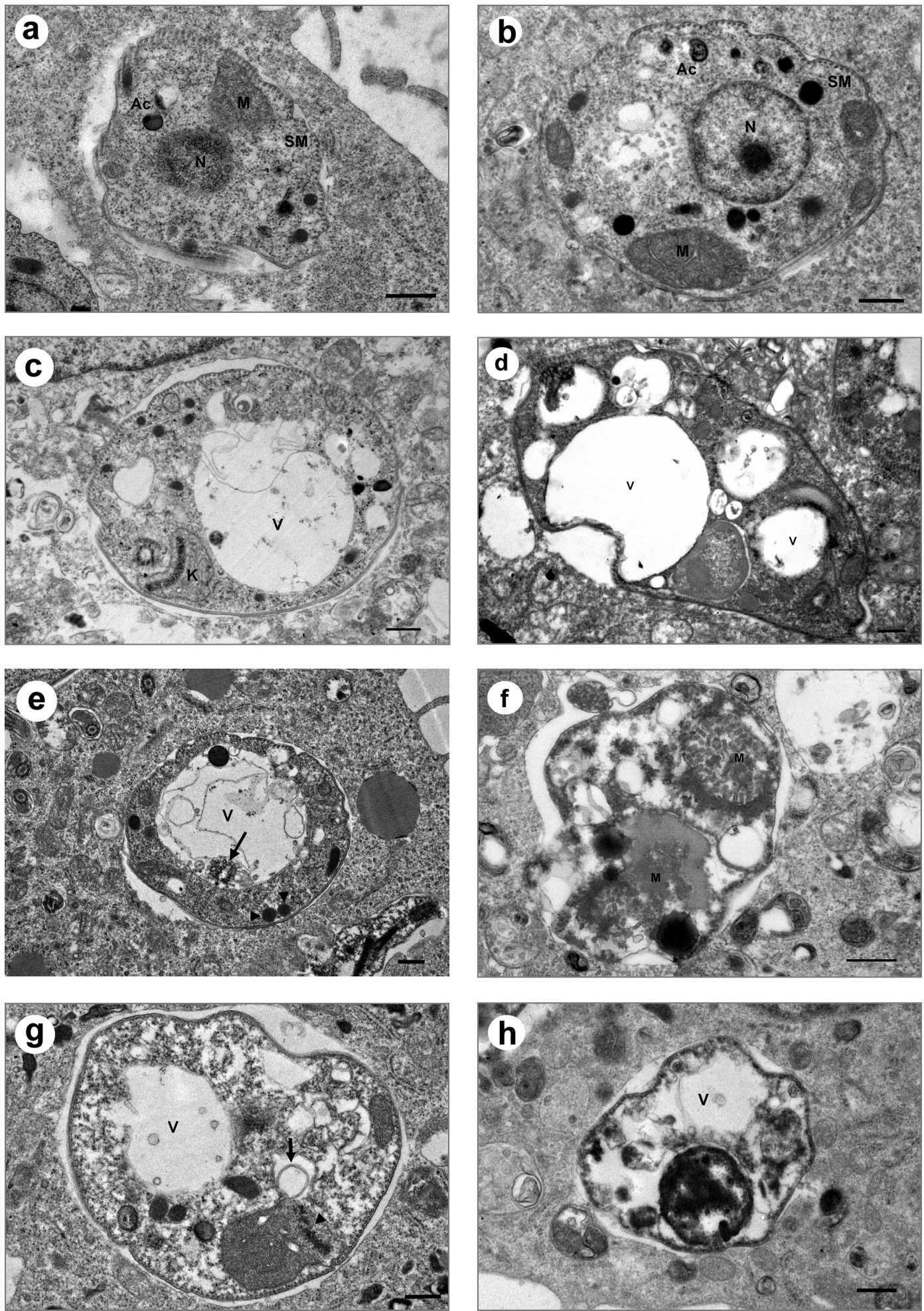

Fig. 2. Ultrastructural alterations caused by a $4 \mathrm{hr}$-incubation of intracellular amastigotes with $10 \mu \mathrm{M}$ allopurinol, azathioprine and 5-fluorouracil after 24 hrs. (a, b) Controls: L. donovani (a) and L. infantum (b) intracellular amastigotes showing the nucleus (N), a layer of subpellicular microtubules (SM), the mitochondrion (M) and acidocalcisomes (AC). (c, d) Allopurinol: extensive vacuolization (V) with a normal kinetoplast (K) in L. donovani (c) and L. infantum (d) in the presence of the control drug. (e, f) Azathioprine: presence of lipid bodies (arrowheads), multivesicular material inside the flagellar pocket (arrows) in L. donovani (e) and disruption of the mitochondrion (M) in L. infantum (f) amastigotes. (g, h) 5-fluorouracil: autophagosome-like structures (arrow) and alteration of the kinetoplast (arrowhead) in L. donovani (g) and cell autolysis in L. infantum (h). Bar: $0.5 \mu \mathrm{m}$ 
Table I. Cytotoxicity of the analogues for J744-A1 macrophages. The means $\pm \mathrm{SD}$ of the $\mathrm{IC}_{50}$ values are given in $\mu \mathrm{M}$

\begin{tabular}{|c|c|c|c|c|}
\hline & & $24 \mathrm{hr}$ & $48 \mathrm{hr}$ & $72 \mathrm{hr}$ \\
\hline & Amphotericin B & $5.4 \pm 1.1$ & $3.6 \pm 0.7$ & $2.2 \pm 0.4$ \\
\hline \multirow[t]{6}{*}{ Controls } & Glucantime & $42.4 \pm 0.4$ & $>100$ & $>100$ \\
\hline & Allopurinol & $>100$ & $>100$ & $>100$ \\
\hline & 6-mercaptopurine & $>100$ & $>100$ & $>100$ \\
\hline & Cytarabine & $>100$ & $>100$ & $>100$ \\
\hline & Azathioprine & $>100$ & $>100$ & $>100$ \\
\hline & 5-fluorouracil & $>100$ & $>100$ & $>100$ \\
\hline
\end{tabular}

the other compounds displayed $\mathrm{IC}_{50}$ values higher than $100 \mu \mathrm{M}$, glucantime being slightly more toxic (Table I).

In vitro antiproliferative effects on Leishmania donovani and $L$. infantum

\section{Effect on the promastigote stage}

Direct observation of treated promastigotes by light microscopy showed that they lost their motility. Generally, the compounds' inhibitory effect began at the onset of incubation and after $4 \mathrm{hr}$, a difference in sensitivity could be seen between the strains tested, cytarabine being much more active on the canine L. infantum strain (Fig. 1). There was a significant difference between the two strains after $24 \mathrm{hrs,}$ except for azathioprine. Among the control compounds, allopurinol displayed a far better inhibitory activity than glucantime.

\section{Effect on the amastigote stage}

The antiproliferative effect on amastigotes is shown in Table II. In the control cultures, the parasite load amounted approximately to $60 \%$ at $24 \mathrm{hrs}$ and was still $40 \%$ at $72 \mathrm{~h}$. Among the control drugs, allopurinol was the most potent. On both strains, only 5-fluorouracil and azathioprine were inhibitory, whereas the other compounds were ineffective.

Table II. Effect of the analogues against intracellular L. donovani and $L$. infantum amastigotes. The means $\pm \mathrm{SD}$ of the $\mathrm{IC}_{50}$ values are given in $\mu \mathrm{M}$. The infected macrophages were incubated for $4 \mathrm{hrs}$ with the drugs and their inhibitory activity was evaluated after $72 \mathrm{hrs}$

\begin{tabular}{lcc}
\hline \multicolumn{1}{c}{$\mathbf{I C}_{\mathbf{5 0}}(\boldsymbol{\mu M})$} & L. donovani & L. infantum \\
\hline Glucantime & $26.7 \pm 8.5$ & $1.0 \pm 0.1$ \\
Allopurinol & $17.0 \pm 0.7$ & $0.6 \pm 0.3$ \\
Azathioprine & $1.9 \pm 0.7$ & $2.8 \pm 1.4$ \\
6-mercaptopurine & $>100$ & $>100$ \\
Cytarabine & $>100$ & $>100$ \\
5-Fluorouracil & $1.0 \pm 0.2$ & $3.0 \pm 1.3$ \\
\hline
\end{tabular}

Effect of azathioprine and 5-fluorouracil on the amastigote ultrastructure

Since 5-fluorouracil and azathioprine were the most effective against this stage, their effect on the morphology of the intracellular amastigotes was studied (Fig. 2). In the treated amastigote-infected cells, the intracellular parasites displayed severe structural damages. In the presence of allopurinol, an extensive vacuolization of the cytoplasm was observed and in addition, the presence of lipid bodies and mitochondrial swelling with loss of the matrix content was found in azathioprine-treated cultures. In 5- fluorouracil-treated amastigotes, autophagosome-like structures were observed together with alterations of the kinetoplast structure that led to autolysis of the parasites.

\section{Discussion}

The combination of Glucantime and of the purine analogue allopurinol is considered so far to be the most effective therapy for canine leishmaniasis (Solano-Gallego et al. 2011), which is not the case in humans, due to pharmacokinetic properties (Croft and Olliaro 2011). Infection of the mammalian host depends on the metacyclic promastigote form and it has been reported recently that metacyclogenesis is highly influenced by nucleosides, especially adenosine (Serafim et al. 2012). In purine stress conditions, Leishmania parasites maximize salvage by up-regulating specific enzymes and transporters of the purine metabolic pathway (Carter et al. 2010, Martin et al. 2014). Although resistance mechanisms to purine starvation allow parasite survival, this pathway is a target of choice for rational drug design (De Koning et al. 2005, Vincent and Barrett 2015). For instance, the nucleoside analogues immucillins reduced in vitro the replication of $L$. infantum intramacrophagic amastigotes (Freitas et al. 2015). The pyrimidine nucleoside analogue 5-fluorouracil is converted in vivo into the active metabolite 5fluoroxyuridine monophosphate (F-UMP) that incorporates into RNA, thereby inhibiting cell growth. Despite the ability of Leishmania parasites to both biosynthesise and salvage pyrimidines from their host environment, $L$. donovani mutants deficient in the enzyme of pyrimidine salvage LdUPRT, were hypersensitive to high concentrations of uracil, 5- fluorouracil 
and 4-thiouracil (Soysa et al. 2013). We thus chose to test some purine and pyrimidine analogues for their ability to inhibit in vitro the multiplication of both stages of $L$. donovani and $L$. infantum.

The tested compounds and the control drug allopurinol showed a low cytotoxicity on macrophages. The higher cytotoxicity was found with amphotericin $\mathrm{B}$, which was not subsequently used, and to a much lesser extent, with glucantime.

The antiproliferative activity tests on promastigotes evidenced that cytarabine and the control drug allopurinol were the most effective after $24 \mathrm{hrs}$, compared to Glucantime. It is interesting to note that, except for cytarabine and allopurinol, the promastigotes of $L$. donovani were generally more sensitive than those of $L$. infantum. Azathioprine is a prodrug metabolized into 6-mercaptopurine and in humans, glutathione S-transferase (GST) is involved (Eklund et al. 2006). In promastigotes, involvement of the trypanothione-based redox metabolism could be possible (Leroux and Krauth-Siegel 2015). It is not surprising that its effect seemed delayed compared to the other analogues, while the effect of its active metabolite 6mercaptopurine was observable at the beginning of incubation. The pyrimidine analogue 5-fluorouracil was generally more active on $L$. donovani than on the $L$. infantum canine strain, while it was the contrary for cytarabine.

When the analogues were incubated with intracellular amastigotes, cytarabine and 6-mercaptopurine were ineffective, whereas 5-fluouracil and azathioprine displayed the highest activity, with $\mathrm{IC}_{50}$ values lower than $10 \mu \mathrm{M}$. The possibility that the inefficient compounds could be metabolized before reaching the intracellular parasite might thus be raised. The inhibitory effect was observable after $72 \mathrm{hrs}$, but incubation for $4 \mathrm{hrs}$ is closer to what happens in vivo and in vitro, where exogenous purines are metabolized within $6 \mathrm{hrs}$ in the culture medium (Lawton et al. 2003). This emphasizes that in vitro inhibition tests should always be performed at first on the amastigote stage, the molecules displaying varying activities according to the insect or the mammalian stages and to their extra- or intracellular status and their respiratory metabolism (Mondal et al., 2014).

Transmission electron microscopy evidenced global alterations of the intracellular amastigotes after treatment with the analogues. Allopurinol produced an intense cytoplasm vacuolization, but in addition, azathioprine and 5-fluorouracyl triggered also an intense mitochondrial swelling and with the latter, alterations of the kinetoplast possibly due to degradation of the DNA structure. The presence of autophagosome-like structures could be related with a remodelling process of damaged organelles by autophagy, resulting in an autolysis process.

\section{Conclusion}

The present work confirms the deleterious effect of nucleoside analogues against two Leishmania strains, human and ca- nine. The pyrimidine analogue 5-fluorouracil was effective against both stages. Conversely, the deleterious effect of azathioprine was more visible in amastigotes, due to its probable rapid metabolism in the host cell. Our results confirm that in vitro tests of potential drug candidates should be made on intracellular amastigotes, since the observed effects can vary according to the culture conditions and the free or intracellular status of the parasites.

\section{References}

Balasegaram M., Ritmeijer K., Lima M.A., Burza S., Ortiz Genovese G., Milani B., et al. 2012. Liposomal amphotericin B as a treatment for human leishmaniasis. Expert Opinion on Emerging Drugs, 17, 493-510. DOI: 10.1517/14728214.2012.748036

Carter N.S., Yates P.A., Gessford S.K., Galagan S.R., Landfear S.M., Ullman B. 2010. Adaptive responses to purine starvation in Leishmania donovani. Molecular Microbiology, 78, 92107. DOI: $10.1111 /$ j.1365-2958.2010.07327.x

Croft S.L., Olliaro P. 2011. Leishmaniasis chemotherapy-challenges and opportunities. Clinical Microbiology and Infection, 17, 1478-1483. DOI: 10.1111/j.1469-0691.2011.03630.x

Croft S.L., Sundar S., Fairlamb A.H. 2006. Drug resistance in leishmaniasis. Clinical Microbiological Reviews, 19, 111126. DOI: $10.1128 / C M R .19 .1 .111-126.2006$

De Koning H.P., Bridges D.J., Burchmore R.J.S. 2005. Purine and pyrimidine transport in pathogenic protozoa: from biology to therapy. FEMS Microbiology Reviews, 29, 987-1020. DOI: 10.1016/j.femsre.2005.03.004

Dorlo T.P.C., Balasegaram M., Beijnen J.H., de Vries P.J., 2012. Miltefosine: a review of its pharmacology and therapeutic efficacy in the treatment of leishmaniasis. Journal of Antimicrobial Chemotherapy, 67, 2576-2597. DOI: 10.1093/ $\mathrm{jac} / \mathrm{dks} 275$

Eklund B.I., Moberg M., Bergquist J., Mannervik B. 2006. Divergent activities of human glutathione transferases in the bioactivation of azathioprine. Molecular Pharmacology, 70, 747-754. DOI: 10.1124/mol.106.025288

Farca A.M., Miniscalco B., Badino P. Odore R., Monticelli P., Trisciu oglio A., Ferroglio E. 2012. Canine leishmaniosis: in vitro efficacy of miltefosine and marbofloxacin alone or in combination with allopurinol against clinical strains of Leishmania infantum. Parasitology Research, 110, 2509-2513. DOI: $10.1007 / \mathrm{s} 00436-011-2792-7$

Freitas E.O., Nico D., Guan R., Meyer-Fernandes J.R., Clinch K., Evans G.B., et al. 2015. Immucillins Impair Leishmania (L.) infantum chagasi and Leishmania (L.) amazonensis multiplication in vitro. PLoS One, 10, e0124183. DOI: 10.1371/journal. pone. 0124183

Lawton P., Hejl C., Mancassola R., Naciri M., Petavy A.F. 2003. Effects of purine nucleosides on the in vitro growth of Cryptosporidium parvum. FEMS Microbiology Letters, 226, 39-43. DOI: 10.1016/S0378-1097(03)00555-X

Leroux A.E., Krauth-Siegel R.L. 2015. Thiol redox biology of Trypanosomatids and potential targets for chemotherapy. Molecular and Biochemical Parasitology, DOI: 10.1016/j.molbiopara.2015.11.003

Martin J.L., Yates P.A., Soysa R., Alfaro J.F., Yang F., Burnum-Johnson K.E., et al. 2014. Metabolic reprogramming during purine stress in the protozoan pathogen Leishmania donovani. PLoS Pathogens, 10, e1003938. DOI: 10.1371/journal.ppat. 1003938 
Mondal S., Roy J.J., Bera T. 2014. Generation of adenosine tri-phosphate in Leishmania donovani amastigote forms. Acta Parasitologica, 59, 11-16. DOI : 10.2478/s11686-014-0203-9

Monge-Maillo B., López-Vélez R. 2013. Therapeutic options for visceral leishmaniasis. Drugs, 73, 1863-1888. DOI: 10.1007/s40265-013-0133-0

Pachioni J. de A., Magalhães J.G., Lima E.J.C., Bueno L. de M., Barbosa J.F., de Sá M.M., Rangel-Yagui C.O., 2013. Alkylphospholipids - a promising class of chemotherapeutic agents with a broad pharmacological spectrum. Journal of Pharmacy and Pharmaceutical Sciences, 16, 742-759. DOI: $10.18433 / \mathrm{J} 3990 \mathrm{~V}$

Serafim T.D., Figueiredo A.B., Costa P.A.C., Marques-da-Silva E.A., Gonçalves R., de Moura S.A.L., et al. 2012. Leishmania metacyclogenesis is promoted in the absence of purines. PLoS Neglected Tropical Diseases, 6, e1833. DOI: 10.1371/journal.pntd.0001833

Received: January 31, 2017

Revised: April 12, 2017

Accepted for publication: April 26, 2017
Solano-Gallego L., Miró G., Koutinas A., Cardoso L., Pennisi M.G., Ferrer L., et al. 2011. LeishVet guidelines for the practical management of canine leishmaniosis. Parasites \& Vectors, 4, 86-102. DOI: 10.1186/1756-3305-4-86

Soysa R., Wilson Z.N., Elferich J., Forquer I., Shinde U., Riscoe M.K., et al. 2013. Substrate inhibition of uracil phosphoribosyltransferase by uracil can account for the uracil growth sensitivity of Leishmania donovani pyrimidine auxotrophs. Journal of Biological Chemistry, 288, 2995429964. DOI: $10.1074 /$ jbc.M113.478826

Sundar S., Chakravarty J. 2013. Leishmaniasis: an update of current pharmacotherapy. Expert Opinion on Pharmacotherapy, 14, 53-63. DOI: 10.1517/14656566.2013.755515

Vincent I.M., Barrett M.P. 2015. Metabolomic-based strategies for anti-parasite drug discovery. Journal of Biomolecular Screening, 20, 44-55. DOI: 10.1177/1087057114551519 